\title{
Challenges and impact of COVID-19 on teledermatology practice in providing continued care to patients in a time of disarray
}

\author{
Ghazala Butt ${ }^{1}$, Mahwash Rana², Muhammad Uzair ${ }^{3}$
}

${ }^{1}$ Department of Dermatology, KEMU/Mayo Hospital, Lahore, Pakistan, ${ }^{2}$ Department of Dermatology, Continental Medical College, Lahore, Pakistan, ${ }^{3}$ Department of Dermatology, KMU-Institute of Medical Sciences Kohat, Pakistan

Corresponding author: Muhammad Uzair, MD, E-mail: waziruzair1@gmail.com

\begin{abstract}
Background: The coronavirus disease 2019 (COVID-19) pandemic has significantly affected all areas of life in most countries. Telehealth has gained importance during this era of social distancing, including teledermatology (TD). The purpose of this survey was to determine the challenges and impact of COVID-19 on TD practice in providing continued care to patients by dermatologists in Pakistan. Methods: A questionnaire comprised of fifteen questions was created with Google Forms and distributed to dermatologists practicing in various cities of Pakistan via WhatsApp or e-mail, then the data was collected. Results: A total of 81 dermatologists from various cities of Pakistan responded to the survey, among whom about two thirds reported a positive experience with TD, yet some had issues in communication gaps and breaches of confidentiality, and the majority considered it unequal to in-person visits. Conclusion: TD plays an important role during the COVID-19 pandemic as a simple, time-saving procedure allowing social distancing with good patient satisfaction.
\end{abstract}

Key words: impact; COVID-19; teledermatology

\section{INTRODUCTION}

Coronavirus disease 2019 (COVID-19) initially reported in Wuhan, China, in December 2019 was declared a pandemic by the WHO in March 2020 [1]. It has rapidly spread all over the world and now affects 214 countries worldwide, with over 103.2 million cases and 2.2 million deaths [2]. Pakistan has also suffered, with 547,648 confirmed cases and 11,746 deaths, and has entered the second peak phase [2].

COVID-19 has affected almost every aspect of life and has had huge social, economic, and healthcare impact [3]. One of the most effective measures for reducing the rapid spread of COVID-19 in the community is social distancing, as demonstrated by several researchers [4]. The minimal recommended distance for effective social distancing by the WHO is $1 \mathrm{~m}$ [5], whereas other researchers are in favor of even greater distances [6]. Healthcare workers are particularly at risk of exposure to COVID-19 during routine occupational activities and, in turn, may themselves become sources of viral dissemination in the community [7]. Therefore, hospitals and healthcare systems all over the world have postponed all non-urgent face-to-face consultations and elective surgery procedures to reduce healthcare related spread of the infection [8,9]. Similarly, in-person dermatological consultations have been significantly reduced worldwide, including in Pakistan $[9,10]$.

The global decrease in clinical consultations has led to increasing dependence on telehealth procedures [11]. Telehealth utilizes modern communication technologies during medical consultations and teaching while maintaining distance [12]. Teledermatology (TD) is

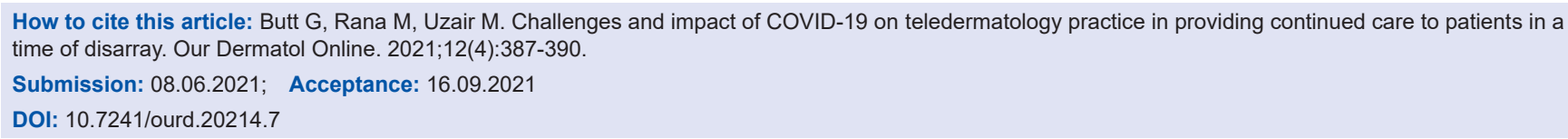


the application of telehealth services in dermatological practice [13]. Since diagnosis in dermatology is greatly dependent on visual examination, it is highly suitable for telemedicine practice [14]. TD is able to ensure continued provision of basic dermatological care for patients while decreasing COVID-19 risk for both healthcare practitioners and patients. Furthermore, TD helps triage patients who need in-patient visits for complete cutaneous examination and any relevant procedures such as skin biopsy [15].

Several researchers worldwide have reported the trend of shifting dermatology consultations to TD platforms with positive outcomes [16]. Some facilities have even transitioned completely to e-health during peak COVID-19 infection rates [17]. There have been numerous studies on the use of TD for providing dermatological care with the focus on dermatologists' experience and patient outcomes relevant to their skin disorder $[13,18,19]$. In Pakistan, since the imposition of countrywide lockdown in April 2020, which was partially lifted later, dermatology outpatient departments have not resumed full operation, especially in government sectors. Furthermore, private consultations have also been reduced significantly during the COVID-19 pandemic [10]. However, no study has been done on TD practice in Pakistan during COVID-19 according to our knowledge. The objective of our study was to assess the use of TD and the challenges faced by dermatologists from all over Pakistan during the COVID-19 pandemic.

\section{METHODS}

A questionnaire comprised of fifteen questions was created with Google Forms and distributed to dermatologists practicing in various cities of Pakistan via WhatsApp or e-mail, then the data was collected in July 2020. The last date of data collection was July 25, 2020 .

\section{RESULTS}

The survey was distributed among Pakistan Association of Dermatologists (PAD)-registered dermatologists and 81 of them responded. Among these 50 were females and 31 males. These ranged from professors to postgraduate trainees and medical officers. The vast majority (75\%) belonged to Punjab, followed by KPK, with only 3 from Islamabad, and 2 and 1 from
Balochistan and Sindh, respectively. None belonged to Gilgit-Baltistan.

Only 4 respondents (5\%) had never done TD till the date of the survey, while the majority had used it to varying degrees. WhatsApp was the medium used almost exclusively, with only rare instances of Facebook or audio calls.

Most participants had a positive experience with TD and reported an increase in TD consultations since the pre-COVID era. Many, however, considered TD visits unlike face-to-face consultations. The responses were divided over the level of communication comfort and many practitioners considered such communication insufficient compared to inperson visits. Technologic malfunction was highly uncommon.

\section{DISCUSSION}

COVID-19-related social distancing has led to a significant decrease in non-urgent face-to-face consultations worldwide, including in Pakistan [8-10]. Simultaneously, there has been a shift in favor of telehealth $[10,11]$, and this is likely to continue till the pandemic either ends or a safe and effective vaccine is available for the general population.

Our survey assessed the experience of dermatologists in Pakistan and the drawbacks and difficulties that they had experienced while practicing TD during the COVID-19 pandemic. It was noted that almost two thirds of our respondents were females while males comprised one third. This ratio may be due to the fact that female doctors tend to prefer dermatology as a field of medicine in Pakistan. Most of the participants (77.8\%) were professorial staff or consultant dermatologists. This may be due to private practice by consultants, which has shifted partly or wholly to online services during the pandemic. Punjab comprised the main chunk, probably due to the home territory of the surveyed.

It was noted that only a handful (5\%) had never practiced TD till the date of the survey, while most $(95 \%)$ had used it with varying frequencies. This is comparable to studies from the U.S., and India, where telehealth practice by dermatologists during COVID-19 comprised $86.5 \%$ and $88.5 \%$, respectively $[18,19]$. However, a German survey revealed that only $38.8 \%$ of dermatology practices offered TD, although there 
had been a four-fold increase since the pre-COVID times [9].

WhatsApp was the predominant platform used by practitioners in our survey (90\%) and very rarely audio calls or Facebook were employed. This agrees with studies from all over the world, where the use of WhatsApp as a telehealth tool, including TD, during the pandemic has been advocated $[20,21]$. Similarly, researches even from before the appearance of COVID-19 had favored WhatsApp for teleconsultations [22], and this has become even more relevant in the present social distancing era. The widespread use of WhatsApp as a means of communication by the general population globally even prior to the pandemic [23] and the ease of video calling and image sharing and minimal data charges have made it the medium of choice for TD practice in Pakistan.

Most of our participants had a positive experience with TD. Two thirds considered it to improve access to healthcare, help to save time, and keep patients safe during the pandemic. Andrees et al. also consider $\mathrm{TD}$ as a time-effective platform that may supplement or replace traditional visits [24]. About three quarters found it easy and simple to use, although only a half reported their experience as pleasant. The discomfort felt by some of the surveyed may be attributed to the lack of routine use of TD before the COVID-19 pandemic. $60 \%$ of the practitioners felt that teleconsultations improved their productivity. More than two thirds reported an increase in TD consultations since the pre-COVID era. This agrees with international studies from Germany, the U.S., and India $[9,18,19]$. However, a majority $(76 \%)$ considered TD visits unlike face-toface consultations. Furthermore, the responses were divided over the level of communication comfort and many practitioners considered such communication insufficient compared to in-person visits. Whited, too, reviewed that, although clinicians found TD as comprehensive as physical visits, they had more confidence in clinical visits due to more effective communication [25]. A meta-analysis by Bastola also revealed that the diagnostic accuracy of TD is less than in-person checkups [15]. Similarly, a pre-COVID review found that traditional consultations were diagnostically more effective than TD [26]. Mehrtens et al., on the other hand, found fair diagnostic concordance (68\%) between TD and in-person visits, as did Arzberger et al. $[27,28]$. Meanwhile, a review by Trettel et al. found variable results, with some studies in favor of TD diagnoses and other studies opposing [29].
The clinicians in our survey reported variable patient satisfaction. However, in a recent survey, Ruggerio et al. from Italy found more than $90 \%$ of their patients were satisfied with teleconsultations [30]. Similarly, Mehrtens et al. observed that $82 \%$ of the patients surveyed were satisfied with TD care [27]. The perception of moderate-to-low patient satisfaction by our dermatologists may be due to the novelty of this approach for both patients and caregivers in Pakistan, as well as the feeling of incomplete consultation by the patient when compared to traditional in-person visits.

Our respondents were divided over the perception of the patient's confidentiality breach. Bull et al. from the U.S. also observed that some patients and practitioners had privacy issues with online consultations [31]. This lack of a proper system to maintain patient confidentiality was also pointed out by Wang et al. in their review [32].

Most of our participants experienced uncommon technologic difficulties. The long-standing status of WhatsApp as a popular social media network even before the current pandemic probably accounts for the uncommon technologic malfunctions observed.

Limitations of our study include a relatively small sample size with a predominant representation of the Punjab province. Furthermore, since the relaxation of COVID-19 restrictions, the experiences of the respondents may have diverged from those expressed in our survey. Patient experience regarding the level of privacy and satisfaction may be surveyed independently of the clinician viewpoint. Further studies with larger sample sizes may help to outline the drawbacks and advantages of TD practice in Pakistan in more detail as we enter the second peak phase of COVID-19.

\section{CONCLUSION}

TD has emerged as a time-saving, effective, and easily accessible platform that may continue to provide good healthcare while ensuring the safety of patients and healthcare personnel. Overall, most participating dermatologists in our survey had a good experience regarding the use of TD during COVID-19 restrictions. There are some barriers, such as communication gaps, the patient's confidentiality, and the present lack of clinician confidence compared with a clinical setup. However, it will continue as an important strategy for the provision of outpatient and non-urgent medical 
care to the dermatologically unwell patient in the near future. At the same time, there is a need for formulating and implementing universal guidelines and a structural framework that would safeguard the interests of patients being treated with telehealth as well as those of attending physicians.

\section{Statement of Human and Animal Rights}

All the procedures followed were in accordance with the ethical standards of the responsible committee on human experimentation (institutional and national) and with the 2008 revision of the Declaration of Helsinki of 1975.

\section{Statement of Informed Consent}

Informed consent for participation in this study was obtained from all patients.

\section{REFERENCES}

1. Cucinotta D, Vanelli M. WHO declares COVID-19 a pandemic. Acta Biomed. 2020;91:157-60.

2. WHO Coronavirus Disease (COVID-19) Dashboard Data - Up to date data on pandemic. Last updated: 9:45am CET, 3 February 2021

3. Ashraf BN. Economic impact of government interventions during the COVID-19 pandemic: International evidence from financial markets. J Behav Exp Finance. 2020;27:100371.

4. Singley A, Callender Highlander H. A mathematical model for the effect of social distancing on the spread of COVID-19. Spora: J Biomath. 2020;6:40-51.

5. Advice for public. Who.int. https://www.who.int/emergencies/ diseases/novelcoronavirus- 2019/advice-for-public. Published 2020. Accessed 17 October, 2020.

6. Setti L, Passarini F, De Gennaro G, Barbieri P, Perrone MG, Borelli M, et al. Airborne transmission route of COVID-19: Why 2 meters 6 feet of inter-personal distance could not be enough. Int J Environ Res Public Health. 2020;17:2932.

7. Nguyen LH, Drew DA, Graham MS, Joshi AD, Guo CG, Ma W, et al. Risk of COVID-19 among front-line health-care workers and the general community: A prospective cohort study. Lancet Public Health. 2020;5:e475-83.

8. Iacobucci G. Covid-19: All non-urgent elective surgery is suspended for at least three months in England. BMJ. 2020;368:m1106.

9. Elsner P. Teledermatology in the times of COVID-19-A systematic review. J Dtsch Dermatol Ges. 2020;18:841-5.

10. Butt G, Uzair M, Rehman K, Iftikhar U, Naumeri F, Hanif A. Impact of COVID-19 on private practice of dermatology in Pakistan. J Pak Assoc Dermatol. 2020;30:222-8.

11. Weskit J, Fudim M, Cameron B, Gellad ZF, Cho A, Phinney D, et al. Telehealth Transformation: COVID-19 and the rise of Virtual Care. J Am Med Inform Assoc. 2020;27:957-62.

12. Wurm EM, Hofmann-Wellenhof R, Wurm R, Soyer HP. Telemedicine and teledermatology: Past, present and future. J Dtsch Dermatol Ges. 2008;6:106-12.

13. Abbott LM, Miller R, Janda M, Bennett H, Taylor ML, Arnold C, et al. A review of literature supporting the development of practice guidelines for teledermatology in Australia. Aust J Dermatol. 2020;61:e174-83.

14. Düker I, Elsner P. Dermatology in telemedicine. Possibilities and limits. Hautarzt. 2002;53:11.

15. Bastola M, Locatis C, Fontelo P. diagnostic reliability of in-person versus remote dermatology: A meta-analysis. Telemed J E Health. 2021;27:247-50.

16. Mostafa PI, Hegazy AA. Dermatological consultations in the COVID-19 era: Is teledermatology the key to social distancing? An Egyptian experience. J Dermatolog Treat. 2020 Jul 11:1-6.

17. Perkins S, Cohen JM, Nelson CA, Bunick CG. Teledermatology in the era of COVID-19: Experience of an Academic Department of Dermatology. J Am Acad Dermatol (Online). July;83:e43-4.

18. Gorrepati PL, Smith GP. Analysis of availability, types, and implementation of teledermatology services during COVID-19. J Am Acad Dermatol. 2020;83:958-9.

19. Sharma A, Jindal V, Singla P, Goldust M, Mhatre M. Will teledermatology be the silver lining during and after COVID-19? Dermatol Ther. 2020;33:e13643.

20. Jakhar D, Kaul S, Kaur I. WhatsApp messenger as a teledermatology tool during coronavirus disease (COVID-19): from bedside to phone-side. Clin Exp Dermatol. 2020;45:739-40.

21. Villani A, Annunziata MC, Abategiovanni L, Fabbrocini G. Teledermatology for acne patients: How to reduce face-to-face visits during COVID-19 pandemic. J Cosmet Dermatol. 2020;19:1828.

22. Giordano V, Koch H, Godoy-Santos A, Dias Belangero W, Esteves Santos Pires R, Labronici P. WhatsApp messenger as an adjunctive tool for telemedicine: An Overview. Interact J Med Res. 2017;6:e11

23. Montag C, Błaszkiewicz K, Sariyska R, Lachmann B, Andone I, Trendafilov B, et al, Smartphone usage in the $21^{\text {st }}$ century: who is active on WhatsApp? BMC research notes. 2015;8:331.

24. Andrees V, Klein TM, Augustin M, Otten M. Live interactive teledermatology compared to in-person care-a systematic review. J Eur Acad Dermatol Venereol. 2020;34:733-45.

25. Whited JD. Teledermatology research review. Int J Dermatol. 2006;45:220-9.

26. Warshaw EM, Hillman YJ, Greer NL, Hagel EM, MacDonald R, Rutks IR, et al. Teledermatology for diagnosis and management of skin conditions: A systematic review. J Am Acad Dermatol. 2011;64:759-72

27. Mehrtens SH, Shall L, Halpern SM. A 14-year review of a UK teledermatology service: Experience of over 40000 teleconsultations. Clin Exp Dermatol. 2019;44:874-81.

28. Arzberger E, Curiel-Lewandrowski C, Blum A, Chubisov D, Oakley A, Rademaker M, et al. Teledermoscopy in high-risk melanoma patients: A comparative study of face-to-face and teledermatology visits. Acta Derm Venereo. 2016;96:779-84.

29. Trettel A, Easing L, Augustin M. Telemedicine in dermatology: findings and experiences worldwide-A systematic literature review. J Eur Acad Dermatol Venereol. 2018;32:215-24.

30. Ruggiero A, Megna M, Annunziata MC, Abategiovanni L, Scalvenzi M, Tajani A, et al. Teledermatology for acne during COVID-19: high patients' satisfaction in spite of the emergency. J Eur Acad Dermatol Venereol. 2020;34:e662-3.

31. Bull TP, Dewar AR, Malvey DM, Szalma JL Considerations for the telehealth systems of tomorrow: An analysis of student perceptions of telehealth technologies JMIR Med Educ. 2016;2:e11.

32. Wang RH, Barbieri JS, Nguyen HP, Stavert R, Forman HP, Bolognia JL, et al; Group for Research of Policy Dynamics in Dermatology. Clinical effectiveness and cost-effectiveness of teledermatology: Where are we now, and what are the barriers to adoption? J Am Acad Dermatol. 2020;83:299-307.

Copyright by Ghazala Butt, et al. This is an open access article distributed under the terms of the Creative Commons Attribution License, which permits unrestricted use, distribution, and reproduction in any medium, provided the original author and source are credited.

Source of Support: Nil, Conflict of Interest: None declared. 